\title{
Average-Case Competitive Analyses for Ski-Rental Problems
}

\author{
Hiroshi Fujiwara and Kazuo Iwama \\ Graduate School of Informatics, Kyoto University, Kyoto 606-8501, Japan. \\ fujiwara, iwama@kuis.kyoto-u.ac.jp.
}

\begin{abstract}
Let $s$ be the ratio of the cost for purchasing skis over the cost for renting them. Then the famous result for the ski-rental problem shows that skiers should buy their skis after renting them $(s-1)$ times, which gives us an optimal competitive ratio of $2-1 / s$. In practice, however, it appears that many skiers buy their skis before this optimal point of time and also many skiers keep renting them forever. In this paper we show that this behavior of skiers is quite reasonable by using an average-case competitive ratio. For an exponential input distribution $f(t)=\lambda e^{-\lambda t}$, optimal strategies are (i) if $1 / \lambda \leq s$, then skiers should rent their skis forever and (ii) otherwise they should purchase them after renting approximately $s^{2} \lambda(<s)$ times. Thus average-case competitive analyses give us the result which differs from the worst-case competitive analysis and also differs from the traditional average cost analysis. Other distributions and related problems are also discussed.
\end{abstract}

Suppose that the costs of renting and purchasing skis are $\$ 100$ (per ski tour) and $\$ 1000$, respectively. Then the well-known result for the ski-rental problem says that skiers should rent skis for the first nine $(=(1000-100) / 100)$ times and should purchase them when going to the tenth. This strategy gives us an optimal competitive ratio (= 1.9 for this price setting) [Kar92]. In practice, however, many skiers seem to buy their skis earlier than this optimal point of time or to keep renting them forever. In this paper we show that this common behavior of skiers is quite reasonable from a theoretical point of view, using average-case competitive analyses.

Average-case analyses for the competitive ratio of online problems have rarely appeared in the literature, although they are quite popular in other areas of algorithms. The reason is probably as follows: An online problem can be regarded as a game between an online player and an adversary. The adversary selects the input which attacks weakest points of the online player's strategy. To make this possible, the adversary must have the freedom of selecting inputs without any restriction. Note that average-case analyses assume some input distribution, which is public to the online player. The adversary of course has to follow the distribution and this can be a significant restriction against the adversary's freedom, or even destroy the essence of online games. This seems to be a common perception of most researchers who have had a negative attitude against such a model. 
In this paper we reconsider this common perception, and claim that averagecase competitive analyses occasionally provide us with even more interesting results compared with the conventional worst-case analysis. Our problem here is the ski-rental problem already mentioned. The costs of renting and purchasing skis are denoted by 1 and $s$, respectively. An online algorithm for this problem is determined completely by deciding how many $(=k)$ times the skier should rent skis before buying them, and therefore such an algorithm is denoted by $A(k)$. We use a continuous model merely because of the ease of calculation. It should be noted that similar results are obtained by using an equivalent discrete model and we often use the terminology of the discrete model, such as "in each occasion of going to a ski tour." Let $\operatorname{ALG}(k, t)$ and $\operatorname{OPT}(t)$ denote the cost of the online algorithm and the cost of the optimal offline algorithm, respectively, where $t$ is the total number of times the skier goes skiing. Apparently,

$$
\begin{aligned}
\operatorname{ALG}(k, t) & = \begin{cases}t, & 0 \leq t \leq k, \\
k+s, & k<t,\end{cases} \\
\operatorname{OPT}(t) & =\min (s, t) .
\end{aligned}
$$

The worst-case competitive ratio for the algorithm $A(k)$ is denoted by $\max _{t}(\operatorname{ALG}(k, t) / \operatorname{OPT}(t))$.

Lemma 1. The following strategy provides an optimal worst-case competitive ratio of 2: the skier should purchase skis after renting s times.

Now let $f(t)$ be a probability density function for the input distribution. Then we can define the average-case competitive ratio as

$$
c(k)=\boldsymbol{E}\left[\frac{\operatorname{ALG}(k, t)}{\mathrm{OPT}(t)}\right]=\int_{0}^{\infty} \frac{\operatorname{ALG}(k, t)}{\mathrm{OPT}(t)} \cdot f(t) d t .
$$

As the function $f(t)$, we use a so-called exponential distribution defined by

$$
f(t)=\lambda e^{-\lambda t} \quad(\lambda>0)
$$

As mentioned before, this distribution means that on each occasion the skier continues to go skiing with probability $1-\lambda$ and quits skiing with $\lambda$. Note that its mean value $\int_{0}^{\infty} t \lambda e^{-\lambda t} d t$ is equal to $1 / \lambda$, which shows how many times in total the skier goes skiing on average.

We calculate (3) for two regions $0<k \leq s$ and $s<k$, for which the value of $c(k)$ is denoted by $c_{1}(k)$ and $c_{2}(k)$, respectively. For $0<k \leq s$, one can obtain

$$
\begin{aligned}
c_{1}(k) & =1-e^{-\lambda k}+(k+s) \int_{k}^{s} \frac{1}{t} \lambda e^{-\lambda t} d t+\frac{k+s}{s} e^{-\lambda s} \\
& =1-e^{-\lambda k}+\lambda(k+s)(E i(-\lambda s)-E i(-\lambda k))+\frac{k+s}{s} e^{-\lambda s},
\end{aligned}
$$

where $\operatorname{Ei}(-x)=-\int_{x}^{\infty}\left(e^{-t} / t\right) d t$, called the exponential integral, cannot be expressed as an elementary function [MUH56]. On the other hand, for $s<k$, we 
can obtain

$$
c_{2}(k)=1+\frac{1}{\lambda s} e^{-\lambda s}-\left(\frac{1}{\lambda s}-1\right) e^{-\lambda k} .
$$

Optimal strategies of the online player differ depending on whether $1 / \lambda \leq s$ or $1 / \lambda>s$. Note that $1 / \lambda \leq s$ means that the average number of total ski tours is equal to or less than the cost of purchase.

Theorem 1. The following strategy provides an optimal average-case competitive ratio for the exponential input distribution $f(t)=\lambda e^{-\lambda t}$ : (i) If $1 / \lambda \leq s$, then the skier should rent skis forever. (ii) Otherwise, the skier should purchase skis after renting $k_{0}$ times, where $k_{0}$ satisfies $s^{2} \lambda-s / 10<k_{0}<s^{2} \lambda$.

Acknowledgments We are grateful to Yasuo Okabe and Gerhard Woeginger for their useful comments.

\section{References}

[Kar92] R. Karp, On-line algorithms versus off-line algorithms: how much is it worth to know the future?, Proc. IFIP 12th World Computer Congress, Vol. 1, pp. 416-429, 1992.

[MUH56] S. Moriguchi, K. Udagawa and S. Hitotsumatsu, Mathematics Formulas I, Iwanami Shoten, Tokyo, 1956. 